\title{
A pseudo-iatrogenic case of medial clavicular fracture
}

\author{
Tibor Antonius Johannes Poelmann • \\ Heleen Muriel Staal · Willem Jacob Willems
}

Received: 30 May 2007/ Accepted: 4 December 2007/Published online: 3 January 2008

(C) Springer-Verlag 2007

\begin{abstract}
Medial fractures are the least common type of clavicular fracture $(2-10 \%)$. The patient is a 29 -year-old gynaecology resident with hyper-laxity and sternoclavicular instability. The latter had been surgically stabilized with Dacron ${ }^{\circledR}$ tape, which eroded the bone causing an usura. Acute right shoulder pain occurred 10 years later. CT revealed medial clavicular stress fracture. After 4 weeks of conservative management, internal fixation followed. Five months postoperatively the patient performed all activities without pain. In this patient the weakened medial clavicle due to usura clearly played a role in both the site and nature of the fracture. Furthermore, CT is essential in arriving at the correct diagnosis.
\end{abstract}

Keywords Clavicle - Stress fracture $\cdot$ CT ·

Occupational history · Usura

\section{Introduction}

Medial fractures are the least common type of clavicular fractures $(2-10 \%)$. This is confirmed by a biomechanical study by Harnroongroj et al. [1]. A compression load along the axis of the S-shaped clavicle produces a middle third fracture [1]. A type III fracture is a fracture through the

T. A. J. Poelmann · H. M. Staal · W. J. Willems

Department of Orthopaedic Surgery,

Onze Lieve Vrouwe Gasthuis, P.O. Box 95500,

1090 HM Amsterdam, The Netherlands

e-mail: w.j.willems@olvg.nl

T. A. J. Poelmann ( $\square)$

Transvaalstraat 78c, 1092 HP Amsterdam,

The Netherlands

e-mail: tiborpoelmann@ hotmail.com medial part of the clavicle, with an incidence of $2-10 \%$ [2-4]. Postachini et al. [3] found an incidence of $2.1 \%$ for type III fractures. In reality this is probably somewhat higher because medial clavicular fractures may easily go unrecognized on plain radiographs. Throckmorton et al. [4] found an incidence of $9.3 \%$ for medial clavicular fractures. In their series, however, CT scanning was liberally used.

This case report describes a case of medial clavicular fracture, emphasizing the role of diagnostic imaging including CT.

\section{Case report}

The patient is a 29-year-old female gynaecology resident with known hyper-laxity. Ten years before she intensively practised gymnastics and even made it to the national selection. The complaints started with a sternoclavicular dislocation subsequent to a back hand manoeuvre during tennis which was surgically corrected.

The operation involved stabilization of the sternoclavicular joint with an autologous graft palmaris longus tendon and fixation of the clavicle to the first rib with a Dacron $^{\circledR}$ tape. The patient was free of symptoms for 9 years and played high level tennis during this period. After 9 years, however, progressive sternoclavicular pain and instability upon elevation occurred.

X-ray-examinations demonstrated an usura involving one third of the clavicular diameter, probably caused by the Dacron ${ }^{\circledR}$ tape (Fig. 1).

A surgical reconstruction of the sternoclavicular joint was performed a year after the recurrence of symptoms. First the Dacron $^{\circledR}$ tape was removed and then a double-stranded autologous semitendinosus tendon was used to stabilize the sternoclavicular joint. This was combined with a 


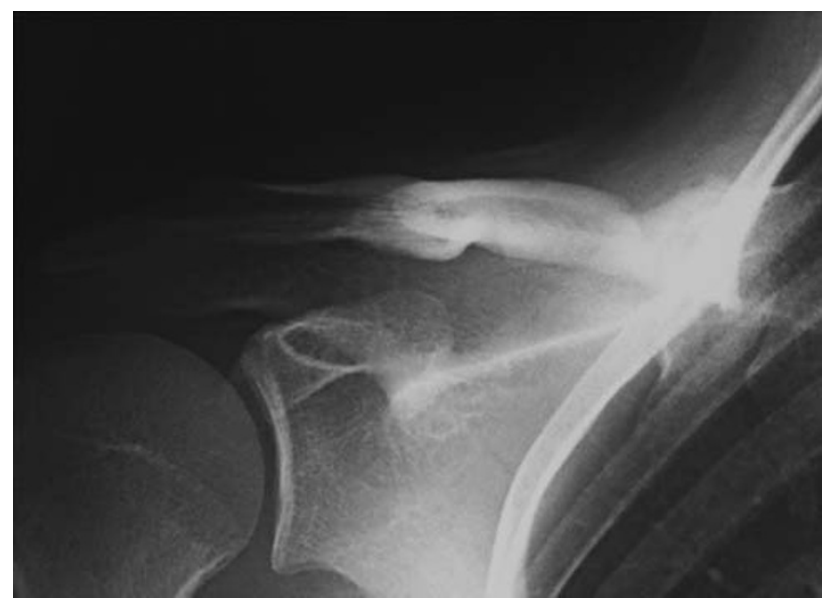

Fig. 1 Radiograph of our patient revealing an usura on the right medial clavicle

transposition of the sternocleidomastoid muscle to the clavicle. Initially this gave good results, with the patient being able to work again, including operating and assisting labour.

A year later the patient returned with acute pain in the right shoulder, radiating to the sternoclavicular joint, which was not preceded by trauma. She was unable to work.

CT-scan demonstrated a stress fracture of the medial clavicle (Figs. 2, 3) due to the usura, which in turn was probably caused by the Dacron ${ }^{\circledR}$ tape that had been used in the first operation.

Four weeks of conservative management did not have any effect. Internal fixation with plates, screws and autograft iliac crest bone followed.

Three months postoperatively resumption of physically exerting work (deliveries) was initiated. The patient was

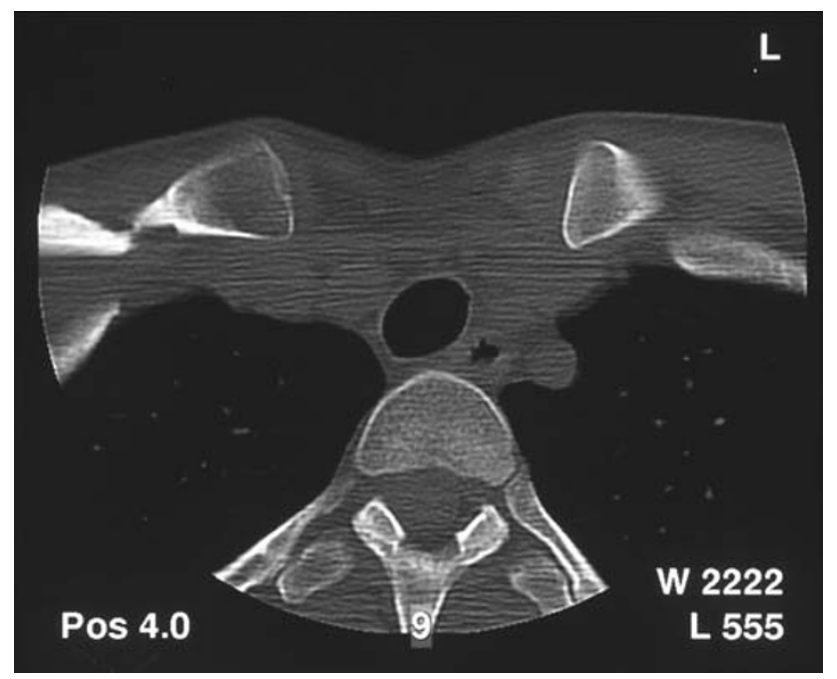

Fig. 2 CT-scan of our patient revealing right medial clavicular stress fracture

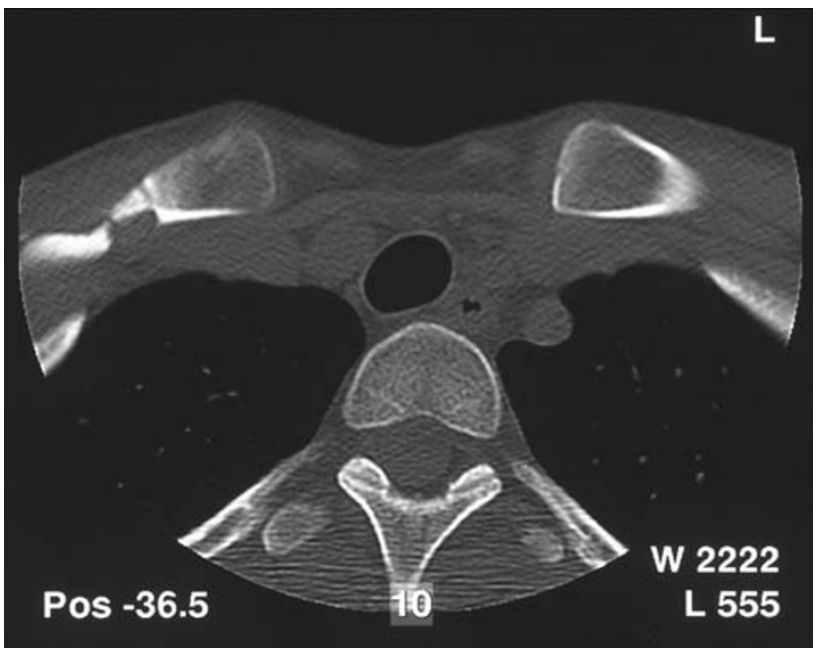

Fig. 3 CT-scan of our patient revealing right medial clavicular stress fracture

able to do everything again without pain 5 months postoperatively.

\section{Discussion}

To our knowledge, literature on medial clavicular fractures is scarce, but some case reports and one series have been published.

The above mentioned series, is a review of all 57 medial clavicular fractures treated at a tertiary trauma center over a 5 year period. The injuries were, in contrast to our case, typically the result of vehicular trauma accompanied by multisystem trauma and with a high associated mortality rate. Interestingly, $22 \%$ of the medial clavicular fractures in this series were only visualized on CT and not on plain radiographs [4].

A striking report is a case of medial clavicular fracture due to a nervous tic.

The report describes a 27-year-old man with a painful tumour-like lesion over the medial clavicle, which was found to be a stress fracture caused by a nervous tic resulting from mental stress. It was hypothesized that the continuous contractions of the sternocleidomastoid muscle produced this stress fracture [5].

A medial clavicular stress-fracture in a 50-year-old cable-maker was caused by holding a heavy drum on his left shoulder while tightening a belt, an action repeated several hundred times per day [6].

Another medial clavicular stress fracture, in this case in a 12-year-old boy, was not caused by strenuous athletic activity. Biopsy was prompted by the suspicion of malignancy or infection. Further historical data, obtained after biopsy, indicated that stressful academic activity 
(carrying a large number of heavy books under his left arm every day) had been the cause of the stress fracture [7].

What these cases have in common is that they all illustrate the necessity for proper history taking, especially occupational history taking, particularly when stress fracture is a differential diagnostic possibility. By doing so, diagnostic tools to exclude diagnoses like malignancies may prove unnecessary.

Furthermore, a wide range of activities of a repetitive nature have been identified as causes of medial clavicular stress fracture. This implies detailed questions regarding (repetitive) activities during the day are necessary.

Two cases of medial clavicular non-union, initially thought to be chronic anterior sternoclavicular dislocations, demonstrate that pseudo-dislocation cannot be distinguished from true-dislocation by physical examination and plain radiographs.

In order to elucidate the nature of the lesion, CT scan should be performed on all patients with suspected or established injuries of the sternoclavicular joint [8].

A case comparable to this report is a case of non-union of a clavicle fracture in which the Dacron ${ }^{\circledR}$ loop had eroded the bone and acted as a stress riser contributing to the trauma. The Dacron ${ }^{\circledR}$ was initially used to stabilise the acromioclavicular joint. Interposition of the Dacron ${ }^{\circledR}$ prevented union of the fracture [9].

Nowadays Dacron ${ }^{\circledR}$ should no longer be used for operative stabilizations of joints. Autologous tendons and muscle transposition used as stabilizers do not have the disadvantage of erosion and worked well in our patient.

\section{Conclusion}

In our case of medial clavicular fracture, arriving at the correct diagnosis was challenging, partly due to the low incidence and partly because the diagnosis can be confused with sternoclavicular dislocation.

With a symptomatic painful medial clavicle, it is advisable to perform $\mathrm{CT}$ in order to rule out a fracture.

Acknowledgments Thanks are due to Mattia Adriano Egidio Valente, MSc.

\section{References}

1. Harnroongroj T, Tantikul C, Keatkor S (2000) The clavicular fracture: a biochemical study of the mechanism of clavicular fracture and modes of the fracture. J Med Assoc Thai 83:663-667

2. Allman F (1967) Fractures and ligamentous injuries of the clavicle and its articulations. J Bone Joint Surg A49:774-784

3. Postachini F, Gumina S, Santis De P, Albo F (2002) Epidemiology of clavicle fractures. J Shoulder Elbow Surg 5:452-456

4. Throckmorton T, Kuhn J (2007) Fractures of the medial end of the clavicle. J Shoulder Elbow Surg 16:49-54

5. Yamada K, Sugiura H, Suzuki Y (2004) Stress fracture of the medial clavicle secondary to nervous tic. Skeletal Radiol 33:534-536

6. Peebles C, Sulkin T, Sampson M (2000) "Cable-maker's clavicle":stres fracture of the medial clavicle. Skeletal Radiol 29:421-423

7. Kaye J, Nance E, Green N (1982) Fatigue fracture of the medial clavicle: an academic rather then an athletic injury. Radiology 144:89-90

8. Brinker M, Simon R (1999) Pseudo-dislocation of the sternoclavicular joint. J Orthop Trauma 13:222-225

9. Dust W, Lenczner E (1989) Stress fracture of the clavicle leading to nonunion secondary to coracoclavicular reconstruction with Dacron ${ }^{\circledR}$. Am J Sports Med 17:128-129 